\title{
Uso de antibióticos en México: revisión de problemas y políticas
}

\author{
Anahí Dreser, MD, MSc, ${ }^{(1,2)}$ Veronika JWirtz, PhD, ${ }^{(2)}$ Kitty K Corbett, PhD, MPH, ${ }^{(3)}$ Gabriela Echániz, PhD. ${ }^{(4)}$
}

\section{Dreser A, Wirtz VJ, Corbett KK, Echániz G. \\ Uso de antibióticos en México: revisión de problemas y políticas.}

Salud Publica Mex 2008;50 supl 4:S480-S487.

\section{Resumen}

El uso inadecuado de antibióticos representa un riesgo para la salud y un desperdicio de recursos económicos en los servicios de salud.Además, contribuye al aumento de la resistencia bacteriana que, a su vez, incrementa los gastos y la mortalidad por enfermedades infecciosas, por lo que se le considera un grave problema de salud pública.Al respecto, la Organización Mundial de la Salud ha recomendado una serie de estrategias fundadas en las políticas farmacéuticas nacionales. En México, diversos aspectos sobre el uso inapropiado de antibióticos han sido documentados. En respuesta se han desarrollado principalmente intervenciones educativas y gerenciales dirigidas a médicos en servicios públicos de salud, así como programas de vigilancia epidemiológica. La investigación y las intervenciones enfocadas en consumidores, farmacias y el sector privado son escasas. Fundamentalmente, no existe una estrategia nacional sobre antibióticos que se refleje en las políticas farmacéuticas y de salud del país.

Palabras clave: utilización de medicamentos; agentes antibacterianos; farmacorresistencia microbiana; política nacional de medicamentos; México
Dreser A, Wirtz VJ, Corbett KK, Echániz G.

Antibiotic use in Mexico:

review of problems and policies.

Salud Publica Mex 2008;50 suppl 4:S480-S487.

\section{Abstract}

The inappropriate use of antibiotics signifies a risk for individual health and a waste of health resources. It triggers the development of antibiotic resistance, which increases expenditures and mortality related to infectious disease, and is hence considered a serious public health problem. The World Health Organization has thus recommended a series of strategies to be included within national pharmaceutical policies. In Mexico, diverse factors related to the inappropriate use of antibiotics have been documented.While the response has been mainly in the form of educational and managerial interventions directed toward physicians in public health services, as well as epidemiological surveillance, there is a paucity of research and interventions focused on consumers, pharmacies, and the private sector. Fundamentally, a comprehensive national strategy for antibiotics is not incorporated into health and pharmaceutical policies.

Key words: drug utilization; antibacterial agents; drug resistance, microbial; national drug policy; Mexico

(I) Health Services Research Unit, London School of Hygiene and Tropical Medicine. Reino Unido.

(2) Centro de Investigación en Sistemas de Salud, Instituto Nacional de Salud Pública. México.

(3) Faculty of Health Sciences, Simon Fraser University. Canadá.

(4) Centro de Investigaciones sobre Enfermedades Infecciosas, Instituto Nacional de Salud Pública. México.

Fecha de recibido: 9 de agosto de 2007 - Fecha de aceptado: 14 de julio de 2008 Solicitud de sobretiros: Dra.Veronika J Wirtz. Centro de Investigación en Sistemas de Salud, Instituto Nacional de Salud Pública. Av. Universidad 655, col. Santa María Ahuacatitlán. 62508 Cuernavaca Morelos, México. Correo electrónico:vwirtz@correo.insp.mx 
L relevancia que los medicamentos tienen para la salud de la población depende de su buena calidad, accesibilidad y uso adecuado. Sin embargo, se estima que, globalmente, la mitad de los medicamentos se prescriben, se dispensan y se consumen de forma inadecuada. ${ }^{1}$ El uso inapropiado de medicamentos tiene importantes consecuencias adversas tanto para la salud de los individuos como para la economía de las familias y de los servicios de salud. El uso inadecuado de antibióticos es particularmente importante, pues contribuye al desarrollo de resistencia bacteriana, la cual reduce la efectividad de tratamientos establecidos e incrementa los gastos y la mortalidad por enfermedades infecciosas, por lo que se considera un grave problema de salud pública* que demanda respuestas en los planos local, nacional y global. ${ }^{2,3}$

A pesar de que el uso de antibióticos ha sido foco de variada investigación en México, ${ }^{4}$ es poca la información publicada que resuma la situación actual en el país, o bien que describa la respuesta que, desde los sistemas y políticas de salud, se ha dado a esta situación. Este tipo de evidencias es primordial para la formulación y evaluación de políticas. ${ }^{5,6}$ El primer objetivo de este trabajo es caracterizar los problemas que se han documentado sobre el uso de antibióticos en México, así como señalar los vacíos de información existentes, con el fin de identificar necesidades de investigación. El segundo objetivo es analizar el tipo de respuesta que se ha dado a los problemas identificados, y proponer líneas de acción dirigidas a mejorar el uso de antibióticos en la nación.

La información presentada se basa, por un lado, en una revisión de la literatura publicada sobre antibióticos, uso de medicamentos y políticas farmacéuticas en México; por el otro, en una revisión de documentos producidos por organizaciones internacionales sobre el uso apropiado de antibióticos; así como en una revisión de documentos oficiales mexicanos sobre políticas farmacéuticas y de salud, hasta $2006 .^{7}$

\section{Antecedentes}

El uso inadecuado de antibióticos incluye la prescripción excesiva (cuando no está justificada) y la selección in-

\footnotetext{
* El problema de uso inadecuado y resistencia se extiende a los antimicrobianos en general, incluyendo antirretrovirales y medicamentos para tratar la malaria. Sin soslayar su relevancia en México, en el presente trabajo no nos centraremos en estos temas. También se han excluido del análisis el uso de antimocrobianos para tratar la tuberculosis, por tratarse de un régimen terapéutico con características especiales.
}

adecuada de tratamiento (tipo, dosis, curso) por médicos y personal de las farmacias, así como la autoprescripción y falta de adherencia al tratamiento por parte de los consumidores. ${ }^{*}$ El uso de los antibióticos, tanto como de otros medicamentos, está determinado por una complejidad de factores, entre los que se encuentran conocimientos y actitudes respecto de los medicamentos (determinantes micro), organización de los sistemas de salud (determinantes meso); así como factores socio-demográficos, culturales, económicos, políticos y regulatorios (determinantes macro) que conforman el contexto donde ocurre la demanda y oferta de medicamentos. ${ }^{8,9}$ Así, mejorar el uso de los antibióticos requiere no solamente de cambios -simultáneos-en la conducta de los profesionales de la salud y de los pacientes, sino también modificar el contexto en cual éstos interactúan; es decir, lograr cambios en los sistemas y las políticas de salud. ${ }^{2}$

Desde la década de los ochenta, la Organización Mundial de la Salud (OMS) ha promovido el uso racional de medicamentos ${ }^{10} \mathrm{y}$ ha recomendado que este aspecto sea integrado en las políticas nacionales de medicamentos. ${ }^{11}$ La Asamblea Mundial de la Salud (ASM) de 1998 instó a los países miembros a desarrollar acciones dirigidas a mejorar el uso de los antibióticos. En 1998, la Conferencia Panamericana de Resistencia Antimicrobiana en las Américas hizo recomendaciones clave para los países de la región sobre mejoramiento del uso de antibióticos. ${ }^{12}$ En el año 2001, la OMS dio a conocer la Estrategia Global para Contener la Resistencia Antimicrobiana. ${ }^{2}$ En su $60^{\text {a }}$ reunión (2006), la AMS reconoció que no es posible aplicar resoluciones sobre resistencia antimicrobiana sin abordar el problema más amplio del uso irracional de medicamentos en los sectores público y privado, y para ello instó a los países miembros a invertir lo necesario en recursos humanos y financiamiento. ${ }^{13}$ En el cuadro I se presenta un resumen de las recomendaciones internacionales para mejorar el uso de medicamentos en general, y de los antibióticos en particular.

\section{Uso de antibióticos en México}

Los antimicrobianos se encuentran entre los medicamentos que más se venden y se consumen en México: representan un mercado anual de 960 millones de dólares y el segundo lugar en ventas anuales (14.3\%) en

\footnotetext{
* Ciertamente hay otras acciones centrales para lograr contener la resistencia bacteriana, sin embargo, en este trabajo nos enfocamos exclusivamente en el problema del uso de antibióticos en humanos.
} 


\section{Cuadro I}

\section{RECOMENDACIONES INTERNACIONALES SOBRE UNA ESTRATEGIA NACIONAL PARA MEJORAR EL USO DE ANTIBIÓTICOS}

Educación sobre medicamentos

- Educar en fármaco-terapéutica a estudiantes de pregrado en el área de la salud (médicos, enfermeras, dentistas, farmacéuticos), así como a profesionales en servicio, basándose en guías terapéuticas, lista de medicamentos esenciales (cuadro básico) y en la resolución de problemas clínicos.

- Educar a estos grupos sobre los factores que influyen en sus hábitos de prescripción/ dispensación.

- Educar a la población sobre el uso apropiado de medicamentos.

- Educar a profesionales de la salud y a los consumidores específicamente sobre el uso apropiado de antibióticos y el problema de resistencia bacteriana.

- Generar y difundir información independiente sobre medicamentos, útil para profesionales de la salud y para consumidores.
Gestión de medicamentos

en servicios de salud

Desarrollar, promover el uso y actualizar guías de tratamiento nacionales, tomando en cuenta patrones de resistencia bacteriana.

- Desarrollar, promover el uso y actualizar el cuadro básico de medicamentos, a partir de las guías terapéuticas.

- Vigilancia epidemiológica sobre uso de antibióticos y resistencia bacteriana (RB) en los hospitales y en la comunidad. Retroalimentación a encargados de tomar decisiones.

- Establecer un laboratorio nacional de referencia.

- Desarrollar sistemas de supervisión, inspección y retroalimentación para la prescripción.

- En hospitales:

- Establecer comités terapéuticos, definir guías de profilaxis y tratamiento acorde a RB.

- Asegurar el acceso y la calidad de los laboratorios de microbiología, y que la información producida se haga llegar a los prescriptores.
Políticas farmacéuticas

y regulación de medicamentos
- Establecer un comité nacional multidisciplinario para coordinar políticas sobre uso de medicamentos, y un comité nacional para coordinar actividades sobre resistencia bacteriana.

- Limitar el registro de medicamentos a aquellos que cumplan estándares de calidad, seguridad y eficacia.

- Definir límites de la publicidad permitida.

- Restringir la venta de antibióticos sólo con prescripción médica. Excepcionalmente podrán ser dispensados sin receta por profesionales capacitados.

- Establecer requisitos para el ejercicio profesional de prescriptores y dispensadores que incluyan la capacitación continua sobre medicamentos.

- Identificar y eliminar incentivos económicos que promuevan el uso inadecuado de medicamentos.

- Asegurar recursos para la instrumentación y cumplimiento de regulaciones.

- Desarrollar indicadores para monitorear y evaluar el impacto de la estrategia.

Fuente: Referencias 2, 6 y II

farmacias privadas en el país, una proporción mayor cuando se compara con otros países desarrollados o en transición con mercados farmacéuticos grandes ${ }^{14}$ (figura 1). A continuación se caracterizan brevemente los problemas descritos en la literatura sobre prescripción, dispensación y consumo de antibióticos en México.

\section{Prescripción de antibióticos}

La prescripción inadecuada de antibióticos en relación con infecciones respiratorias agudas (IRAS) e infecciones gastrointestinales/diarreicas agudas (EDAS), en el sector público, es el tema más ampliamente documentado sobre uso de medicamentos en México. ${ }^{4}$ Investigaciones realizadas sobre todo durante las décadas de 1980 y 1990, concluyeron que entre 60 y $80 \%$ de los pacientes con IRAS y EDAS recibían antibióticos en servicios primarios de salud públicos ${ }^{15-18}$ y privados ${ }^{19,20}$ del país, cuando en realidad su uso se justificaba tan sólo en 10 a $15 \%$ de los casos. ${ }^{17}$ En contraste, un estudio realizado en un hospital de tercer nivel concluyó que si bien la indicación de antibióticos fue mayoritariamente justificada, la dosis y duración de los tratamientos tendieron a ser incorrectos, lo cual significó un alto riesgo para el desarrollo de resistencia bacteriana. ${ }^{21}$ La prescripción inadecuada de antibióticos para profilaxis quirúrgica también ha sido señalada como un problema importante en este sentido, en ambientes hospitalarios. ${ }^{22}$

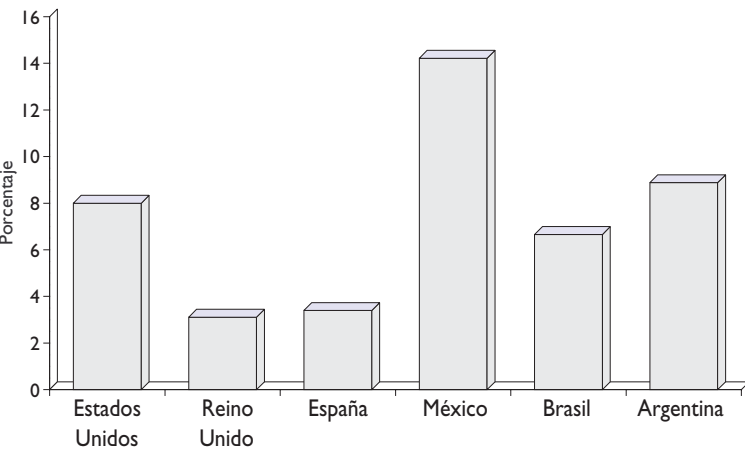

País (mayor a menor mercado farmacéutico)

Fuente: IMS-Health 2005

Figura I. Porcentaje de los antimicrobianos en el MERCADO ANUAL DE MEDICAMENTOS

Algunos de los factores que se han relacionado con la prescripción inadecuada en México son las deficiencias en la educación médica de pre y posgrado; la falta de información independiente sobre medicamentos; la influencia de la información proporcionada por la industria farmacéutica; la percepción de las expectativas de los pacientes en cuanto a recibir medicamentos $y$, 
por último, la prevalencia de patrones de tratamiento incorrectos, pero institucionalizados. ${ }^{23-27}$ Sin embargo, con respecto a la prescripción de antibióticos, hay una escasez de publicaciones recientes, particularmente sobre servicios privados, que permitan caracterizar la situación actual en México, y sus determinantes. Finalmente, otro problema importante, y poco documentado sobre el uso de antibióticos es la falta de adherencia al tratamiento prescrito, que se ha calculado ocurre en alrededor de $55 \%$ de los pacientes con IRAS o EDAS. ${ }^{17}$

\section{Dispensación y autoatención con antibióticos}

En México, donde la autoatención y automedicación representan formas frecuentes de atender los problemas de salud, ${ }^{28,29}$ y donde no se exige la prescripción médica para la venta de antibióticos, el papel que las farmacias privadas desempeñan en el consumo de estos productos es central. Estudios realizados en ese tipo de farmacias, durante la década de 1990, demostraron que los antibióticos eran los medicamentos más vendidos -con y sin prescripción médica-, en particular para tratar IRAS y EDAS. ${ }^{28,30}$ Un estudio concluyó que cerca de $90 \%$ de las compras de antibióticos para EDAS, sin prescripción, en farmacias, eran inadecuadas respecto al tipo, dosis y duración del tratamiento. ${ }^{31}$ Dado que en México no se requiere de un profesional de la salud de tiempo completo en las farmacias, la mayoría de los medicamentos son dispensados por empleados poco capacitados. Estudios más recientes indican que entre 70 y $80 \%$ de las recomendaciones terapéuticas proporcionadas por empleados de farmacias para IRAS, EDAS e infecciones del tracto genito-urinario, mayoritariamente incluyendo antibióticos, son incorrectas. ${ }^{32-34}$ Además, dado que los medicamentos que requieren para su venta prescripción médica -como los antibióticos- no incluyen un inserto informativo, los pacientes que se automedican no pueden acceder a ningún tipo de información sobre las medicinas que consumen. Es poca la información disponible que analiza los determinantes de la dispensación y automedicación con antibióticos.

\section{Calidad y seguridad de los antibióticos}

Otros problemas notificados con respecto a los antibióticos en México son la comercialización de productos de baja calidad, ${ }^{35}$ falsificados ${ }^{36}$ y en combinaciones irracionales (tales como antibióticos más antidiarreicos o analgésicos), ${ }^{30,37}$ lo cual afecta la efectividad del tratamiento e incrementa el riesgo de reacciones adversas y el desarrollo de resistencia bacteriana. No obstante, no hay información científica publicada que exponga, de forma representativa, en todo el país, la calidad y seguridad (incluyendo, por ejemplo, bioequivalencia) de todos los productos antibacterianos comercializados en México.

\section{Consecuencias del uso inadecuado de antibióticos}

Es escasa la información publicada que permita analizar el impacto en el país del uso inapropiado de antibióticos, en términos de gasto en salud, resistencia bacteriana y daños a la salud (reacciones adversas y falla terapéutica). En términos de gastos, se ha informado que el desperdicio de antibióticos debido a la prescripción injustificada y el incumplimiento de tratamientos en IRAS y EDAS representó $11 \%$ del gasto anual en medicamentos de una institución de salud. ${ }^{17}$ En otro estudio se informó que la prescripción inadecuada (de antibióticos bajo control) representó $63 \%$ del gasto anual en antibióticos de un hospital. ${ }^{38}$ Un estudio en farmacias concluye que la prescripción inadecuada representa, con respecto a los tratamientos estándar, un costo adicional por paciente de 3.57 dólares para IRAS y 8.37 dólares para EDAS, ${ }^{39}$ que equivalen a 0.8 y 1.9 , respectivamente, del salario mínimo diario en México. Finalmente, en México se concluyó que la mayor parte $(38 \%)$ de las reacciones adversas a medicamentos reportadas en una institución de salud ${ }^{40}$ se debieron a medicamentos antiinfecciosos particularmente antibióticos.

La creciente resistencia bacteriana en patógenos causantes de infecciones comunitarias e intrahospitalarias es quizás el problema relacionado con el uso inapropiado de antibióticos más notificado en la literatura científica en México. Por ejemplo, las redes regionales de vigilancia epidemiológica estiman que la tasa de resistencia a penicilina del Streptococcus pneumoniae -causante de infecciones comunitarias graves como neumonía y meningitis- es de alrededor de 55\% en México, ${ }^{41-43}$ porcentaje superior a los de otros países de Latinoamérica como Argentina y Brasil. ${ }^{44,45}$ La resistencia bacteriana en patógenos causantes de infecciones respiratorias, entéricas y de vías urinarias en la comunidad también ha sido documentada. ${ }^{46,47}$ En lo tocante a las infecciones intrahospitalarias, se ha notificado la creciente resistencia en patógenos relacionados con una alta incidencia y mortalidad. ${ }^{48-50}$ El uso inadecuado de antibióticos incluso ha sido señalado como un factor de riesgo de mortalidad mayúsculo en unidades de cuidados intensivos. ${ }^{51}$

\section{Intervenciones, regulación y políticas sobre el uso de antibióticos}

\section{Intervenciones educativas y gerenciales}

En México la mayor parte de las intervenciones documentadas para mejorar el uso de antibióticos son 
de carácter educativo y gerencial, y están dirigidas a mejorar la prescripción médica para IRAS y EDAS en servicios de salud públicos. A ese respecto han destacado las intervenciones promovidas por investigadores del Instituto Mexicano del Seguro Social (IMSS) en las décadas de 1980 y 1990.15,16,52 Por otro lado, diversas instituciones de salud han desarrollado y promovido el uso de guías clínicas para mejorar la prescripción. ${ }^{53,54}$ No existen informes recientes que aborden el impacto de estas guías, ni cómo se vincula su desarrollo con información sobre resistencia bacteriana.

En lo tocante al ámbito hospitalario, se ha hecho énfasis en el control y vigilancia de infecciones nosocomiales, donde destaca la Red Hospitalaria de Vigilancia Epidemiológica $^{55}$ (RHOVE, que desde 1997 recaba información sobre resistencia bacteriana de más de 170 hospitales), y la vigilancia de la prescripción de antibióticos. ${ }^{56,57}$ Además, numerosos hospitales participan en redes para vigilar la resistencia bacteriana, como la Red Mexicana para el Estudio de la Resistencia Bacteriana, y los programas internacionales SENTRY y RESIST. No obstante, en un estudio se concluye que el control de calidad en los laboratorios hospitalarios, así como la calidad de los programas de vigilancia de uso de antibióticos, es muy variable. ${ }^{56}$ No hay información disponible sobre el impacto de estos programas de vigilancia en la calidad de la prescripción de antibióticos, ni tampoco acerca de la forma en que se utiliza la información generada para el diseño de políticas institucionales de uso de antibióticos.

En el plano comunitario, desde 1997 se desarrolla un programa de capacitación en el manejo y dispensación de medicamentos en farmacias, para empleados de mostrador, coordinado ahora por la Comisión Federal de Protección contra Riesgos Sanitarios (COFEPRIS). Asimismo, se ha promovido la automedicación responsable a través de campañas informativas y del mejoramiento de la información en el empaque de productos de libre venta. Ninguno de estos programas tiene un enfoque particular en antibióticos, y no se ha evaluado su impacto en el consumo de estos medicamentos.

\section{Regulación y políticas}

En la actual reglamentación en materia de salud en México se encuentran, de forma dispersa, algunos elementos generales relacionados con el interés de lograr un mejor uso de los medicamentos. Si bien la Ley General de Salud (LGS) legisla con solidez el uso de estupefacientes y psicotrópicos, no existe ninguna mención específica sobre antibióticos o resistencia bacteriana en la LGS ni sus reglamentos derivados. Únicamente el artículo 226 de dicha ley señala la regulación de la venta de medicamentos clasificados como grupo IV (donde se incluyen los antibióticos) con prescripción médica; esta regulación, en la práctica, no se hace cumplir. Respecto a las Normas Oficiales Mexicanas (NOM), solamente la NOM sobre tuberculosis (NOM-006-SSA2-1993) menciona la importancia de actualizar tratamientos de acuerdo con patrones de resistencia bacteriana, y el proyecto de NOM sobre infecciones nosocomiales (PROY-NOM-045-SSA2-2005) incluye la regulación del uso de antibióticos dentro de los hospitales.

En cuanto a políticas de salud, en el Plan Nacional de Salud 2001-2006 (PNS) ${ }^{58}$ se señaló la importancia de los problemas de prescripción injustificada de medicamentos y la automedicación, y se propuso una línea de acción para reforzar el consumo razonado de medicamentos. Empero, para ello sólo se señalaron dos metas: promover el uso de genéricos y legislar el uso de pruebas de intercambiabilidad.$^{59}$ La resistencia bacteriana sólo se discutió respecto del tratamiento de la tuberculosis.

Diversos análisis sobre las políticas farmacéuticas en México concluyen que el uso apropiado de medicamentos ha sido el aspecto más relegado; ${ }^{60,61}$ el acceso a medicamentos, el aseguramiento de su calidad y el desarrollo de la industria farmacéutica han constituido las prioridades. El tema del uso apropiado de medicamentos ha sido abordado primordialmente desde una perspectiva normativa, con un enfoque en la seguridad y eficacia de los medicamentos, y no en las conductas de quienes los utilizan. ${ }^{60}$ La nueva iniciativa de política farmacéutica $^{62}$ no menciona el uso apropiado de los medicamentos como uno de sus objetivos; este tema es escasamente abordado en el documento, y la "prescripción razonada" se enfoca primordialmente a aspectos de farmacoeconomía. No se proponen acciones concretas para mejorar el uso de los antibióticos y contener la resistencia bacteriana..$^{63}$

Finalmente, fuera del sector gubernamental, algunas organizaciones no gubernamentales ${ }^{27} \mathrm{y}$ algunas asociaciones profesionales ${ }^{64}$ también han tenido iniciativas para promover el uso más apropiado de los medicamentos en general, y de los antibióticos en particular.

\section{Discusión}

En México, los antibióticos se encuentran entre los medicamentos que más se consumen. La prescripción injustificada con antibióticos, su dispensación inadecuada, y la laxitud en la regulación sobre la venta de medicamentos que permite la automedicación con antibióticos, son algunos de los factores que se han relacionado con este alto consumo. Dadas las limitaciones de la literatura publicada al respecto, y la actual falta de indicadores 
nacionales para monitorear la utilización de los medicamentos, es imposible evaluar de forma integral la situación del uso de antibióticos en el país. Esto impide realizar un análisis comparativo tanto entre las diversas regiones de México como con otros países, y guiar el desarrollo de políticas de medicamentos. Además, no hay trabajos de investigación sobre los determinantes del uso de antibióticos en México, incluyendo las actitudes de los trabajadores de la salud y del público en general sobre el uso de los antibióticos, la influencia de la publicidad y la organización de los servicios de salud. A pesar de que existen variados informes sobre resistencia bacteriana en el país, esta información no ha sido sistematizada y publicada de tal forma que permita caracterizar la situación en el país y guiar la toma de decisiones. Por otra parte, es poca la información publicada sobre los gastos y los daños a la salud asociados al uso inapropiado de antibióticos.

Las iniciativas para mejorar el uso de antibióticos se han centrado en intervenciones educativas y gerenciales para médicos en el sector público, así como en estrategias para vigilar y contener la resistencia bacteriana, generalmente circunscritas al control de las infecciones nosocomiales. Existen grandes vacíos en materia de intervenciones dirigidas al sector médico privado, a los dispensadores en farmacias, a los pacientes y al público en general. El actual marco normativo y las políticas farmacéuticas y de salud no incorporan estrategias sobre el uso apropiado de antibióticos ni la contención de la resistencia bacteriana, como se recomienda internacionalmente.

Sin duda, el desarrollo e implementación de estrategias orientadas a racionalizar el uso de los medicamentos no es fácil. Algunas de las barreras son: complejidad en la regulación del sector privado; otras prioridades nacionales, como el acceso a medicamentos en el sector público; falta de recursos para instrumentar intervenciones complejas intersectoriales; intereses económicos del sector farmacéutico, e intereses gremiales. ${ }^{5,65-67} \mathrm{Sin}$ embargo, el tema de uso de antibióticos y resistencia bacteriana ha sido capaz de llegar a la agenda política de varios países cuyos parlamentos han discutido y aprobado medidas al respecto, como es el caso del Reino Unido ${ }^{68}$ y de Chile. ${ }^{69}$ El problema de la escasa regulación sobre el uso de antibióticos en México también tiene implicaciones para las naciones vecinas: las bacterias resistentes no reconocen límites territoriales. La frontera de México con los Estados Unidos de América (EUA) es particularmente importante, dada su porosidad para el movimiento de personas y medicamentos. ${ }^{70}$ Ambos países podrían beneficiarse con el trabajo conjunto sobre estrategias para mejorar el uso de antibióticos. Al respecto, los EUA han lanzado ya algunos programas nacionales y estatales dirigidos a poblaciones hispano parlantes. $^{71}$

Aunque hay grandes carencias de información, este análisis de la situación actual, junto con las recomendaciones internacionales sobre el tema, permite sugerir acciones clave dentro de una estrategia nacional dirigida a mejorar el uso de antibióticos en México:

- Crear un comité nacional multidisciplinario e intersectorial encargado de diseñar, monitorear y evaluar una estrategia amplia dirigida a mejorar el uso de antibióticos.

- Fomentar la investigación y la documentación sobre patrones de consumo de antibióticos y sus determinantes, así como sistematizar la información generada por redes de vigilancia de resistencia, con el fin de generar información útil para la toma de decisiones.

- Vincular el funcionamiento y la información de guías terapéuticas, patrones de susceptibilidad bacteriana, el cuadro básico y la educación médica de pre y posgrado.

- Evaluar estrategias para hacer cumplir la ley en cuanto a la dispensación de antibióticos en farmacias sólo con prescripción médica, y acotar la publicidad sobre antibióticos.

- Desarrollar e instrumentar una campaña informativa en los medios de comunicación, dirigida al público general, sobre el uso adecuado de antibióticos.

- Crear indicadores que permitan el monitoreo y rigurosa evaluación de las intervenciones.

En conclusión, existen recomendaciones claras en el plano internacional para lograr un mejor uso de los antibióticos. En México, es prioritario que las políticas farmacéuticas y de salud aborden el problema, con el fin de mejorar la calidad de atención, contener la resistencia bacteriana y disminuir el gasto público y privado en salud. El inicio de un nuevo gobierno en México representa una ventana de oportunidad para introducir este tema en la discusión política y consolidar una estrategia nacional sobre antibióticos, identificando al país como un líder regional en esta iniciativa tan importante para el futuro bienestar de nuestras poblaciones.

\section{Agradecimientos}

Se agradece a los integrantes de la línea de investigación Medicamentos en Salud Pública, del INSP, por sus sugerencias para este trabajo. Anahí Dreser realiza estudios de doctorado en la London School of Hygiene and Tropical Medicine con una beca del Consejo Nacional de Ciencia 
y Tecnología de México (167955) y la agradecida supervisión del Dr. Stuart Anderson. La colaboración con Kitty Corbett fue posible gracias a una beca Fulbright-García Robles y un President's Research Grant de la Simon Fraser University.

\section{Referencias}

I.World Health Organization. The world medicines situation 2004. Geneva:World Health Organization, 2004.

2. World Health Organization. Global strategy for containment of antimicrobial resistance. Geneva:World Health Organization, 200I. 3. Wise R, Hart T, Cars O, Streulens M, Helmuth R, Houvinen P, et al. Antimicrobial resistance is a major threat to public health [editorial]. $\mathrm{Br}$ Med J 1998;317:609-610.

4. Wirtz V], Reich MR, Leyva-Flores R, Dreser A. Medicines in Mexico, 1990-2004: Systematic review of research on access and use. Salud Publica Mex 2008;50 suppl 4:S470-S479.

5. World Health Organization. Comparative analysis of national drug policies in 12 countries. Second workshop, Geneva 10-13 June 1996. Geneva:World Health Organization, 1997.

6. World Health Organization.WHO policy perspectives on medicines. Promoting rational use of medicines: core components. Geneva:World Health Organization, 2002.

7. Dreser A.Antibiotics in Mexico:An analysis of problems, policies and politics. Protocolo de Investigación, Tesis de Doctorado. London School of Hygiene and Tropical Medicine, Londres, 2006.

8. Avorn J, Solomon DH. Cultural and economic factors that (mis)shape antibiotic use: the nonpharmacologic basis of therapeutics. Ann Intern Med 2000;133:128-135.

9. Radyowijati A, Haak H. Improving antibiotic use in low-income countries: an overview of evidence on determinants. Soc Sci Med 2003;57:733-744

10.World Health Organization. The rational use of drugs. Report of the conference of experts, Nairobi, 25-29 November 1985. Geneva:World Health Organization, 1987.

II.World Health Organization. How to develop and implement a national drug policy. 2nd ed. Geneva:World Health Organization, 200I.

12. Pan American Health Organization. Prevention and control of antimicrobial resistance in the Americas. PAHO/HCP/HCT//39/99. Washington: Pan American Health Organization, 1999.

13. Organización Mundial de la Salud. Uso racional de los medicamentos: progresos realizados en la aplicación de la estrategia farmacéutica de la OMS. Informe de la Secretaría, EBI 18/6. Ginebra: Organización Mundial de la Salud, 2006.

14. International Marketing System-Health. IMS Retail Drug Monitor - April: International Marketing System-Health, 2005. [consultado el I3 de abril de 2008] Disponible en: http://www.imshealth.com.

15. Gutiérrez G, Guiscafre H, Bronfman M, Walsh J, Martinez H, Muñoz $\mathrm{O}$. Changing physician prescribing patterns: evaluation of an educational strategy for acute diarrhea in Mexico City. Med Care 1994;32:436-446. 16. Pérez-Cuevas R, Guiscafre H, Muñoz O, Reyes H,Tome P, Libreros V, et al. Improving physician prescribing patterns to treat rhinopharyngitis. Intervention strategies in two health systems of Mexico. Soc Sci Med 1996;42:1 I85-1194.

17. Reyes H, Guiscafre H, Muñoz O, Pérez-Cuevas R, Martínez H, Gutiérrez G.Antibiotic noncompliance and waste in upper respiratory infections and acute diarrhea.J Clin Epidemiol 1997;50:1297-1304. 18. Libreros V, Guiscafre H,Tome P, Reyes H, Pérez-Cuevas R, Gutiérrez $G$, et al. Patrones de prescripción terapéutica en diarrea e infecciones respiratorias agudas en dos instituciones de salud: SS e IMSS. Gac Med Mex 1992; 128:505-552.

19. Bojalil R, Guiscafre H, Espinosa P, Martínez H, Palafox M, Romero G, et al.The quality of private and public primary health care management of children with diarrhoea and acute respiratory infections in Tlaxcala, Mexico. Health Policy Plan 1998; 13:323-331.

20. Peláez-Ballestas I, Hernández-Garduño A,Arredondo-García JL, Viramontes-Madrid JL,Aguilar-Chiu A. Use of antibiotics in upper respiratory infections on patients under 16 years old in private ambulatory medicine. Salud Publica Mex 2003;45:159-164.

21. García-Rubí E, Sierra-Madero JG, Ponce De León-Rosales S. Uso de antibióticos en la consulta externa del Instituto Nacional de la Nutrición "Salvador Zubirán". Rev Invest Clin 1991;43: | |3-1 18.

22. Mendez-Lopes M, Díaz-Ramos R, Ramírez-Velasco J, Miranda-Novales M. Uso de antimicrobianos profilácticos en cirugía en un hospital pediátrico de tercer nivel. Enfermedades Infecciosas y Microbiología 2005;25. [consultado el I3 de abril de 2008] Disponible en :http://www. amimc.org.mx/revista/2005/25-I/uso_antimicrobiano.htm 23. Corral-Terrazas M, Martínez H, Flores-Huerta S, Duque LM, Turnbull B, Levario-Carrillo M. Creencias y conocimientos de un grupo de médicos sobre el manejo de la alimentación del niño con diarrea aguda. Salud Publica Mex 2002;44:303-3।4.

24. Duran-González L, Becerra-Aponte J, Franco F, Kravzov-jinich J,VisoGurovich F, Frenk-Mora J. Uso del cuadro básico de medicamentos en el primer nivel de atención. Salud Publica Mex 1990;32:543-55I. 25. Duran-González LL, Frenk-Mora J, Becerra-Aponte J. La calidad de la conducta prescriptiva en atención primaria. Salud Publica Mex 1990;32:181-191.

26. Reyes $\mathrm{H}$. Efectividad de una intervención educativa basada en el análisis crítico de la práctica médica, para la atención apropiada en medicina familiar: una propuesta factible y sostenible para la educación médica continua. En: García M, Reyes H,Viniegra L, eds. Las múltiples facetas de la investigación en salud: Proyectos estratégicos del Instituto Mexicano del Seguro Social. México DF: IMSS, 2001.

27.Vicencio-Acevedo D. México: Comité nacional por el uso racional de los medicamentos. Boletín Fármacos 1999;2. [consultado el I3 de abril de 2008] Disponible en: http://www.boletinfarmacos.org/download/feb99.rtf 28. Ángeles-Chimal P, Medina-Flores ML, Molina-Rodríguez JF. Automedicación en población urbana de Cuernavaca, Morelos. Salud Publica Mex 1992;34:554-561.

29. Leyva-Flores R, Kageyama ML, Erviti-Erice J. How people respond to illness in Mexico: self-care or medical care? Health Policy 200I;57:15-26. 30. Calva J, Bojalil R. Antibiotic use in a periurban community in Mexico: a household and drugstore survey. Soc Sci Med 1996;42: I I2I-I I 28.

31. Bojalil R, Calva JJ. Antibiotic misuse in diarrhea. A household survey in a Mexican community.J Clin Epidemiol 1994;47:I47-I56.

32. Kroeger A, Ochoa H,Arana B, Diaz A, Rizzo N, Flores W. Inadequate drug advice in the pharmacies of Guatemala and Mexico: the scale of the problem and explanatory factors. Ann Trop Med Parasitol 2001;95:605-616. 33. Leyva-Flores R, Bronfman M, Erviti-Erice J. Simulated clients in drugstores: prescriptive behaviour of drugstore attendants. J Soc Adm Pharm 2000;17:151-158.

34. Turner AN, Ellertson C, Thomas S, Garcia S. Diagnosis and treatment of presumed STIs at Mexican pharmacies: survey results from a random sample of Mexico City pharmacy attendants. Sex Transm Infect 2003;79:224-228.

35. Karlage KL, Myrdal PB. Comparison of three pharmaceutical products obtained from Mexico and the United States: a case study. Drug Dev Ind Pharm 2005;31:993-1000.

36. Secretaría de Salud. Comunicado de Prensa No. 23I. La COFEPRIS, con mayores facultades en materia de productos "milagro". México, DF: Secretaría de Salud, 2005. [Consultado el 13 de abril de 2008] Disponible en: http://www.salud.gob.mx/ssa_app/noticias/datos/2005-05-04_I382.html 
37. Comité Terapéutico y Farmacológico de México. Propuesta de un formulario abierto para un programa de atención médica dirigida en México. Salud Publica Mex 1999;41:27-41.

38. Navarrete-Navarro S, Ávila-Figueroa C, Medina-Cuevas F, SantosPreciado Jl.Vigilancia y costos relacionados con la prescripción de antimicrobianos en un hospital pediátrico. Gac Med Mex 1999;135:383-389. 39. Flores W, Ochoa H, Briggs J, Garcia R, Kroeger A. Economic costs associated with inadequate drug prescribing: an exploratory study in Chiapas, Mexico. Acta Trop 2003;88:57-68.

40. Hernández-Santillán D, Rodríguez-Betancourt L,Vasso-Gutiérrez L. Farmacovigilancia IV: la experiencia institucional. Rev Med IMSS 2005;43:257-266.

4I. Echániz A,Velázquez M, Carnalla B, Soto N, Solórzano S, Pérez M, et al. Antimicrobial susceptibilities and capsular types of invasive Streptococcus pneumoniae in children in Mexico City. Microb Drug Resist 1997 3:I53-I56. 42. Hortal M, Lovgren M, de la Hoz F,Agudelo Cl, Brandileone MC, Camou T, et al.Antibiotic resistance in Streptococcus pneumoniae in six Latin American countries: 1993-1999 surveillance. Microb Drug Resist 200I;7:39I-40I.

43. Quiñones-Falconi F, Calva JJ, Lopez-VidalY, Galicia-Velazco M, Jimenez-Martinez ME, Larios-Mondragon L. Antimicrobial susceptibility patterns of Streptococcus pneumoniae in Mexico. Diagn Microbiol Infect Dis 2004;49:53-58

44. Camargos P, Fischer GB, Mocelin H, Dias C, Ruvinsky R. Penicillin resistance and serotyping of Streptococcus pneumoniae in Latin America. Paediatr Respir Rev 2006;7:209-214.

45. Castanheira M, Gales AC, Mendes RE, Jones RN, Sader HS. Antimicrobial susceptibility of Streptococcus pneumoniae in Latin America: results from five years of the SENTRY Antimicrobial Surveillance Program. Clin Microbiol Infect 2004; 10:645-65 I.

46. Solórzano-Santos F, Miranda-Novales MG. Resistencia de bacterias respiratorias y entéricas a antibióticos. Salud Publica Mex 1998;40:510-5I6. 47. Hernández-Porras M, Salmeron-Arteaga G, Medina-Santillán R. Microbial resistance to antibiotics used to treat urinary tract infections in Mexican children. Proc West Pharmacol Soc 2004;47:I20-I2I. 48. Velázquez-Meza M,Aires de Sousa M, Echaniz-Avilez G, SolórzanoSantos F, Miranda-Novales G, Silva-Sanchez J, et al. Surveillance of methicillin-resistant Staphylococcus aureus in a pediatric hospital in Mexico City during a 7-year period (1997 to 2003): clonal evolution and impact of infection control.J Clin Microbiol 2004;42:3877-3880.

49. Silva J, Gatica R, Aguilar C, Becerra Z, Garza-Ramos U,Velázquez M, et al. Outbreak of infection with extended-spectrum beta-lactamaseproducing Klebsiella pneumoniae in a Mexican hospital. J Clin Microbiol 200I;39:3193-3196.

50. Alcantar-Curiel D, Tinoco JC, Gayosso C, Carlos A, Daza C, PerezPrado MC, et al. Nosocomial bacteremia and urinary tract infections caused by extended-spectrum beta -lactamase-producing Klebsiella pneumoniae with plasmids carrying both SHV-5 and TLA-I genes. Clin Infect Dis 2004;38(8): 1067-1074.

5I. Zaidi M, Sifuentes-Osornio J, Rolon AL,Vazquez G, Rosado R, Sanchez $M$, et al. Inadequate therapy and antibiotic resistance. Risk factors for mortality in the intensive care unit. Arch Med Res 2002;33:290-294. 52. Guiscafre H, Martínez H, Reyes H, Pérez-Cuevas R, Castro R, Munoz $\mathrm{O}$, et al. From research to public health interventions. I. Impact of an educational strategy for physicians to improve treatment practices of common diseases. Arch Med Res 1995;26:S3I-S39.

53. Reyes H, Pérez-Cuevas R, Trejo-y Pérez J, eds. Guías de práctica clínica para medicina familiar: El Manual Moderno. México: Instituto Mexicano de Seguridad Social, 2004.
54. Instituto Nacional de Salud Pública. Boletín Práctica Médica Efectiva. México, DF: Centro de Información para Decisiones en Salud. Instituto Nacional de Salud Pública.[Consultado el I 3 de abril de 2008] Disponible en: http://bvs.insp.mx/

55. Tapia-Conyer R. Presentación. Infecciones nosocomiales. Salud Publica Méx 1999;4I(sup I):3-4.

56. Benavides-Plascencia L,Aldama-Ojeda AL, Javier Vázquez H.Vigilancia de los niveles de uso de antibióticos y perfiles de resistencia bacteriana en hospitales de tercer nivel de la Ciudad de México. Salud Publica Mex 2005;47:219-226.

57. Medina-Cuevas F, Navarrete-Navarro S, Ávila-Figueroa C, SantosPreciado JI. FARMAC: programa diseñado para vigilar la prescripción de antimicrobianos en hospitales. Gac Med Mex 2000; 136:107-III. 58. Secretaría de Salud. Programa Nacional de Salud 200I-2006. México D.F: Secretaría de Salud, 200I:I36.

59. Secretaría de Salud. Metas del Programa Nacional de Salud 200I-2006. Documento de Seguimiento. México, DF: Secretaría de Salud, 200I:44.

60. Gasman N. Drifting through time: pharmaceutical policies in Mexico. Dev Dialogue 1995; I:224-256.

61.World Health Organization. The world drug situation 1988. Geneva: World Health Organization, 1988.

62. Secretaría de Salud-Comisión Federal para la Protección Contra Riesgos Sanitarios. Hacia una política farmacéutica integral para México. México, DF: Secretaría de Salud-Comisión Federal para la Protección Contra Riesgos Sanitarios, 2005.

63. Leyva-Flores R, Wirtz V, Dreser A, Reich MR. Reseña. Hacia una política farmacéutica integral para México. Salud Pub Mex 2006;48:179-180. 64. Alliance for the Prudent Use of Antibiotics. Guadalajara declaration to combat antimicrobial resistance in Latin America. Guadalajara, Mexico: Alliance for the Prudent Use of Antibiotics, 200I. [Consultado el I 3 de abril de 2008] Disponible en: http://www.tufts.edu/med/apua/Chapters/ Guad/Guadalajara.html.

65. Falkenberg T,Tomson G. The World Bank and pharmaceuticals. Health Policy Plan 2000;15:52-58.

66. Kanji N.Action at country level. En: Kanji N, Hardon A, Harnmeijer JW, Mamdani M,Walt G, eds. Drugs policy in developing countries. London Zed Books Ltd, 1992.

67. Reich MR. Essential drugs: economics and politics in international health. Health Policy 1987;8:39-57.

68. Standing Medical Advisory Committee. The path of least resistance. The Stationery Office. London, 1998. [Consultado el I3 de abril de 2008] Disponible en: http://www.dh.gov.uk/en/Publicationsandstatistics/ Publications/PublicationsPolicyAndGuidance/DH_4009357

69. Bavestrello L, Cabello A, Casanova D. Impacto de medidas regulatorias en la tendencia de consumo comunitario de antibióticos en Chile. Rev Med Chil 2002; 130:1265-1272.

70. Casner PR, Guerra LG. Purchasing prescription medication in Mexico without a prescription. The experience at the border.West J Med 1992;156:512-516.

7I. Centers for Disease Control and Prevention. Campaña para promover el uso correcto de los antibióticos. Centers for Disease Control and Prevention, Atlanta, USA, 2007. [Consultado el I 3 de abril de 2008] Disponible en: http://www.cdc.gov/drugresistance/community/ antibioticos.htm. 